\begin{tabular}{|c|c|c|c|}
\hline Eiszeitalter und Gegenwart & $\mathbf{5 4}$ & $\begin{array}{c}20-35 \\
6 \mathrm{Abb} ., 3 \mathrm{Tab} .\end{array}$ & Hannover 2004 \\
\hline
\end{tabular}

\title{
Das Grönenbacher Feld bei Kempten: Neue Befunde zur Typusregion des Mindelglazials und zur Paareiszeit im bayerischen Alpenvorland
}

\author{
RAIMO BECKER-HAUMANN $^{*)}$
}

Keywords: Neogen, Pleistozän, Mindelglazial, Alpenvorland, Morphostratigraphie, Paläoboden, glazifluviatile Terrassen

Zusammenfassung: Im Illergletscher-Vorland, Bayern, ist das Mindelglazial (drittletzter Eiszeitkomplex des nördlichen Alpenvorlandes) am Grönenbacher Feld definiert. Die vorliegenden Geländebefunde zeigen, dass das Vorkommen stratigraphisch zweigeteilt ist und zu einer stratigraphischen Neubewertung zwingt. Die Untergliederung wird durch in zwei Höhenniveaus liegende glazifluviatile Schmelzwasserterrassen dokumentiert, die an der Wurzel des Grönenbacher Feldes in zwei glaziale Serien übergehen. Sie liegen im Süden des Feldes übereinander und werden von einem Verwitterungshorizont im Mühlbach-Tobel unterteilt. Er deutet auf einen eisfreien Zeitabschnitt mit Ausbildung eines Bodenhorizontes hin.

Das ältere Terrassenniveau (Woringer Schotter) wird dem jüngsten Stadial der im Illergletscher-Vorland dreigeteilten Mindeleiszeit zugerechnet (Mindel 3). Die Existenz des Verwitterungshorizontes, morphologische und flussgeschichtliche Argumente sowie der Verwitterungszustand des jüngeren Schotters (Grönenbacher Schotter) legen nahe, dass dieser jünger als mindel-, aber älter als risszeitlich ist. Damit könnte er der Paareiszeit zuzurechnen sein, die nomenklatorisch eingeführt, jedoch bislang umstritten ist. In vorliegender Arbeit werden die Geländebefunde sowie die Folgerungen für die stratigraphische Zuordnung diskutiert.

"Anschrift des Verfassers: PD Dr. Raimo BeCKerHaumann, Geographisches Institut der Universität zu Köln, Albertus-Magnus-Platz, 50674 Köln
Abstract: The Grönenbacher Feld (gravelplain of Grönenbach) is the type locality of the Mindel glaciation in the Illerglacier Foreland, Bavaria. Recent investigations reveal, that the Grönenbacher Feld is subdivided into two stratigraphic units and that there is need for a revise of the regional stratigraphy. The Grönenbacher Feld consists in two glaciofluvial meltwater terraces in different levels, which grade into moraines. These glacial series are seperated by a weathering horizon, which indicate an ice retreat and a time of soil formation.

The older terrace (Woringer gravel) is tied to the youngest period of the Mindel glaciation (Mindel 3), which is subdivided in the Illerglacier Foreland into three stratigraphic units. By arguments of the morphology, river development and the weathering state of the sediment the lower terrace level (Grönenbach gravel) should be younger than the Mindel glaciation, but older than Riss. Probably it can be tied to the Paar iceage, which is defined in the Illerglacier Foreland, but is still under discussion. In the paper at hand the geological setting of the Grönenbacher Feld is described, and the stratigraphic implications of the field observations are discussed.

\section{Einführung}

Das bayerische Alpenvorland ist bis heute für die stratigraphische Gliederung des Pleistozäns eine überaus wichtige Region. Das liegt zum einen an der langen Tradition geowissenschaftlicher Forschungsarbeiten seit den Pionierleistungen von Albrecht Penck an der Wende vom 19. zum 20. Jahrhundert, die das alpine Vereisungsgebiet weithin bekannt gemacht haben, und 


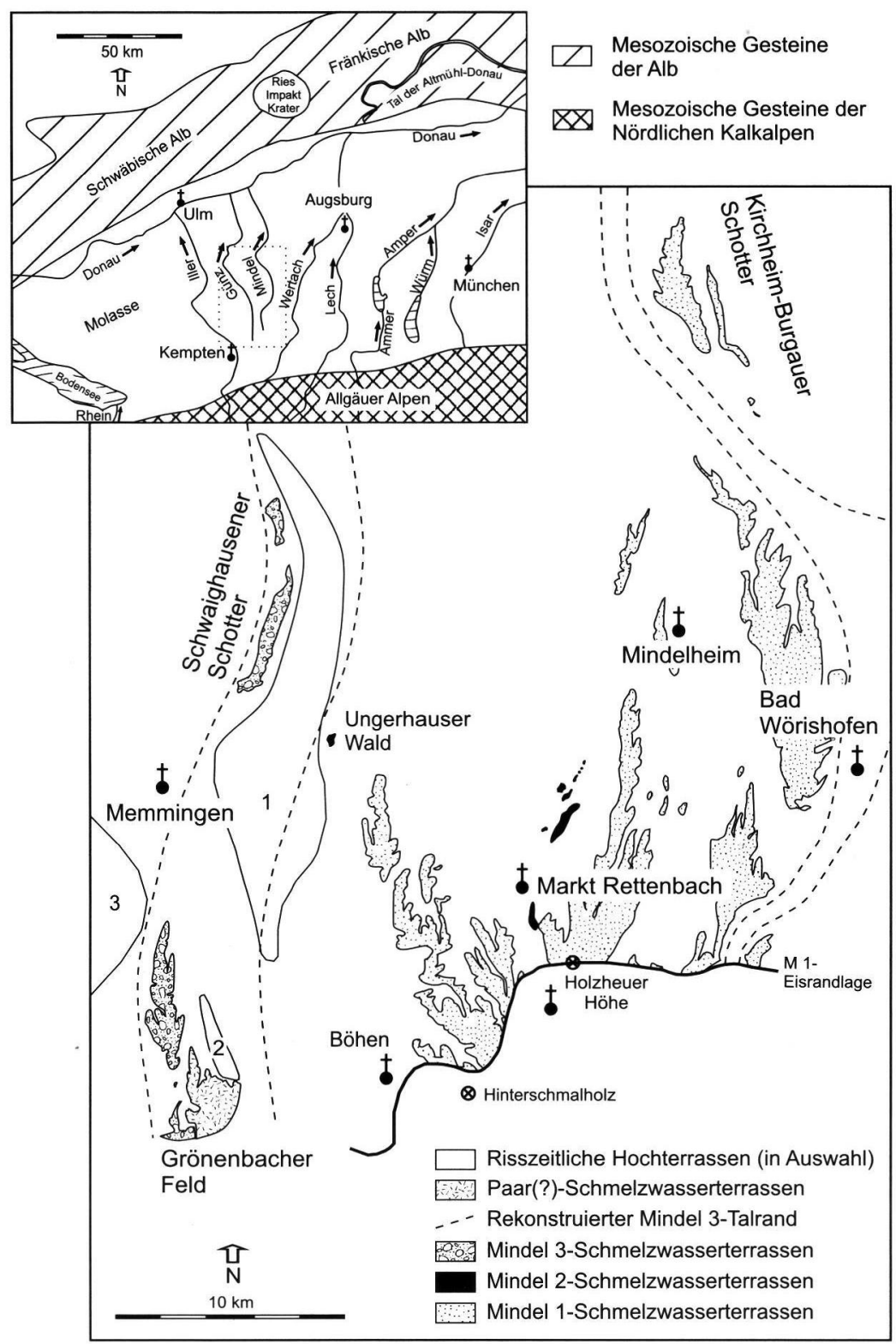

Abb. 1: Übersichtskarte des erweiterten Untersuchungsgebietes im süddeutschen Alpenvorland. Die Schotterverbreitungskarte zeigt die mindelzeitlichen Schmelzwasserablagerungen im Illergletscher-Vorland. Mit gestrichelten Linien ist der rekonstruierte Verlauf des Mindel 3-zeitlichen Abflussbahnen des Illergletschers eingezichnet. Bei den risszeitlichen Hochterrassen im Illertal (Kiessignatur) bedeuten: 1 - Hawanger Feld, 2 - Zeller Hochterrasse, 3 - Hitzenhofer Feld.

Fig. 1: Sketchmap of the area under investigation in the Northern Alpine Foreland, South Germany. The map displays the recent occurrences of Mindel age of the Illerglacier area (grey) and the localities mentioned in the text. Dashed lines indicate the reconstructed valleys of the rivers Günz and Mindel during the Mindel glaciation. The Upper Terraces of Riss age (gravel signature) include the occurrences of: 1 - Hawanger Feld, 2 - Zeller Hochterrasse, 3 - Hitzenhofer Feld. 
zum anderen an der reichhaltigen Gliederung der glazifluviatilen und glazialen Ablagerungen. Ihre gebietsweise weitflächige Erhaltung ermöglicht detailierte Analysen von Einzelgebieten wie auch die Betrachtung großer Regionen im Vorfeld der pleistozänen Alpengletscher, die zur Klärung stratigraphischer, sedimentologischer wie auch paläogeographischer Fragestellungen beitragen. Insbesondere das Illergletscher-Vorland zwischen den heutigen Flüssen Iller, Lech und Donau zeichnet sich durch gut gegliederte unterpleistozäne Flussterrassen aus, von denen eine Anzahl nach Süden in Moränen übergeht und daher eiszeitlicher Entstehung ist. Diese seit Penck \& BRÜCKner (1909) als glaziale Serie bekannte Verknüpfung von Formen und Sedimenten eines Gletscherstandes ist bis heute eine wichtige Grundlage stratigraphischer Überlegungen. Das Illergletscher-Vorland stellt nicht nur ein klassisches Untersuchungsgebiet dar, sondern es wurden hier auch die Typusregionen für wichtige stratigraphische Einheiten definiert.

Die systematische, flächenhafte Neubearbeitung des Illergletscher-Vorlandes (Abb. 1) während der vergangenen zehn Jahre durch die Abteilung Quartärgeologie am Geologischen Institut der Universität zu Köln erbrachte neue Befunde, die auch die Typusregion für das Mindelglazial betreffen. Ein wesentlicher Aspekt ist dabei die Berücksichtigung des gesamten Gletschervorlandes, um Einzelregionen sinnvoll in das Gesamtsystem der stratigraphischen und flussgeschichtlichen Entwicklung einordnen zu können. Dies konnte für die Mindel-Lech-Platte (Becker-Haumann et al. 2001) und die Aindlinger Terrassentreppe (BECKer-Haumann 1995) gezeigt werden. Vor dem Hintergrund der Befunde für das Mindelglazial, die in Becker-Haumann (2002) zusammengefasst sind, wird in vorliegender Arbeit das Grönenbacher Feld behandelt.

Im Illergletscher-Vorland ist bis auf wenige Ausnahmen das Prinzip der Terrassenstaffelung verwirklicht, bei dem die ältesten Niveaus die höchsten Landschaftspositionen einnehmen und die jüngsten Ablagerungen die heutigen Talböden bilden. Die Höhendifferenzen der Terrassen zueinander sind auf Erosionsereignisse in der Zeit zwischen zwei Eisvorstößen zurückzuführen. Die Niveauabstände sind dabei regional unterschiedlich und betragen im IllerLech-Gebiet durchschnittlich 10 bis $15 \mathrm{~m}$. Die von Schaefer (u.a. 1995) angegebenen Abstände von nur zwei bis drei Metern sind sicher nicht in allen Fällen stratigraphisch relevant, sondern auf lokale Faktoren zurückzuführen.

Die Terrassenstratigraphie hat sich im Untersuchungsgebiet bereits seit langem als probate Arbeitsmethode zur Klärung der Ablagerungsreihenfolge von Terrassen durchgesetzt. Die Untersuchung basiert darauf, dass ein Terrassenkörper die Füllung eines vormaligen Tales darstellt, die zwischen den Prozessen Talausräumung und Beendigung der Sedimentation gebildet worden ist. Der zwischen diesen beiden Ereignissen liegende Zeitraum wird durch die Terrassenkörper dokumentiert. In diesem Sinne beschreibt die Terrassenstratigraphie eine Ereignisabfolge, die sich im Iller-Lech-Gebiet mehrfach zyklisch wiederholt hat. Sind die Basis und Oberfläche einer bestimmten Terrasse in ihrer räumlichen Konfiguration bekannt, kann die Geometrie des Paläotales rekonstruiert werden. Neue Möglichkeiten der Auswertung und Darstellung bieten 3D-Untergrundmodelle der Terrassenkörper, mit denen die Geländebefunde in den Kontext der fluviatilen Dynamik sowie der regionalen Geologie gestellt werden (BECKERHaumann im Druck).

Die schotterbedeckten Flächen bei den Orten Woringen und Bad Grönenbach (Abb. 2) werden von PenCK \& BRÜCKNER (1909: 28 und Fig. 3) als Grönenbacher Feld und die pleistozänen Kiese im Rahmen ihres tetraglazialen Systems als "Jüngerer Deckenschotter" mindeleiszeitlichen Alters bezeichnet (Tab. 1). Die zugehörige Moränenverzahnung befindet sich bei der Anhöhe Brandholz. Als nächst äl- 


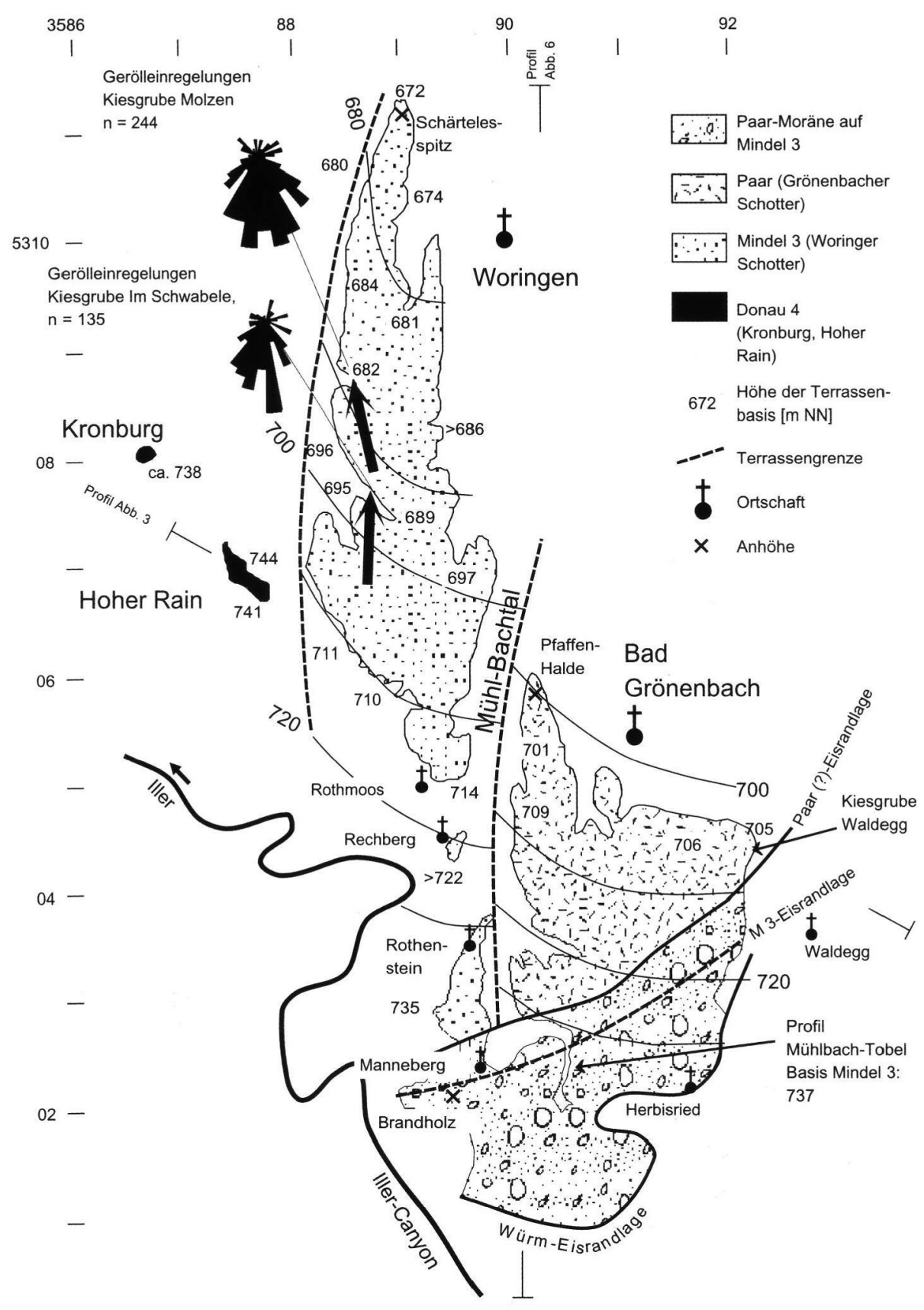

Abb. 2: Schotterverbreitungskarte des Grönenbacher Feldes mit Isohypsen der Terrassenbasis sowie den donauzeitlichen Vorkommen Kronburg und Hoher Rain. Das Profil der (Kgr.) Waldegg ist in Abb. 4, das des Mühlbach-Tobels in Abb. 5 dargestellt.

Fig. 2: Map of the gravel occurrrences of the Grönenbacher Feld showing isohypses of the terrace bases as well as the Donau age gravels of Kronburg and Hoher Rain. The section Waldegg is shown in Fig. 4, the outcrop at the Mühlbach gorge in Fig. 5. 
Tab. 1: Stratigraphische Einstufung und Gliederung des Grönenbacher Feldes nach verschiedenen Autoren. Tab. 1: Age assignments of the Grönenbacher Feld according to several authors.

\begin{tabular}{|l|l|l|}
\hline Autor & $\begin{array}{l}\text { Stratigraphische } \\
\text { Einstufung }\end{array}$ & Gliederung \\
\hline PENCK \& BRÜCKNER (1909) & $\begin{array}{l}\text { Jügerer Deckenschotter } \\
\text { (Mindeleiszeit) }\end{array}$ & ungegliedert \\
\hline EBERL (1930) & Mindeleiszeit & M I, M II \\
\hline SINN (1972) & Mindeleiszeit & ungegliedert \\
\hline SCHAEFER (1973) & Mindeleiszeitengruppe & Schottersysteme I bis $\times$ \\
\hline JERZ et al. (1974) & $\begin{array}{l}\text { Jüngerer Deckenschotter } \\
\text { (Mindeleiszeit) }\end{array}$ & ungegliedert \\
\hline EICHLER \& SINN(1975) & $\begin{array}{l}\text { Mindeleiszeit (als } \\
\text { viertletztes Glazial) }\end{array}$ & ungegliedert \\
\hline LÖSCHER (1976) & Günzeiszeit & ungegliedert \\
\hline HABBE (1985) & $\begin{array}{l}\text { Mindeleiszeit } \\
\text { (ggf. jüngerer Vorstoß) }\end{array}$ & ungegliedert \\
\hline SCHAEFER (1995) & Mindeleiszeitengruppe & Schottersysteme I bis $\times$ \\
\hline
\end{tabular}

tere Einheit gelten die reliktischen Vorkommen an der Kronburg und auf dem Hohen Rain unmittelbar westlich des Grönenbacher Feldes, deren Korrelate PencK \& BRÜCKNer (1909: 30) im „Hochfeld“ (heute als Schrattenbach-Theinselberger Schotter sensu Sinn 1972 oder Böhener Feld sensu Stepp 1981 bezeichnet) sehen. Die Hochterrassen von Zell, Hitzenhofen und Hawangen gelten nach PENCK \& BRÜCKNeR (1909) als einheitlich risszeitlich, wohingegen nach Schaefer (1951: 103) aufgrund differierender Höhen ihrer Oberflächen eine ältere Bildung (Hawanger Feld, Zeller Hochterrasse) von einer jüngeren (Hitzenhofer Feld, Steinbach Hochterrasse) unterschieden wird. EBERL (1930: Tafel I) gliedert das Grönenbacher Feld in einen tiefergelegenen Bereich M I im Süden und einen jüngeren Teil im Woringer Wald, den er als M II bezeichnet. Von Schaefer (1973) werden im Grönenbacher Feld zehn eigenständige Schüttungen unterschieden, die sich mit Höhendifferenzen ihrer Basen von jeweils nur wenigen Metern annähernd von Westen nach Osten untereinander staffeln. Die methodischen Grundlagen dieser Differenzierung sind jedoch nicht generell akzeptiert (Diskussion in Habbe 1986, 1997), so dass diese Einteilung umstritten bleibt. Als ungegliederte mindelzeitliche Akkumulation wird das Grönenba- cher Feld von Sinn (1972), Eichler \& SinN (1975), Jerz et al. (1974) sowie Habbe (1985) angesehen.

Die im Mindeltal erhaltenen Vorkommen (Kirchheim-Burgauer Schotter, Abb. 1) liegen deutlich höher als das Grönenbacher Feld und dessen nördliche Fortsetzung, dem Schwaighausener Schotter. Dies bedeutet ein unterschiedliches Entstehungsalter, da beide Terrassenzüge vom Illergletscher ihren Ausgang nahmen. Die an der Wurzel des Grönenbacher Feldes erhaltene Endmoräne von Brandholz - Manneberg liegt mit ihrer Oberfläche um ca. $50 \mathrm{~m}$ unterhalb der $3 \mathrm{~km}$ östlich verbreiteten Endmoräne bei Böhen, die nach Jerz et al. (1974) beide als mindelzeitlich eingestuft werden. Ein jüngeres Alter als die im Mindeltal erhaltenen Einheiten ist dem Grönenbacher Feld jedoch wegen des Niveauunterschiedes zuzusprechen. Eine Darstellung des Grönenbacher Feldes liegt von HABbe $(1985,1986)$ vor, der von geomorphologischen Befunden ausgehend eine einheitliche Entstehung des Areals konstatiert. Bei den Vorkommen östlich des heutigen Illertales kommt er unter Beibehaltung der Penckschen Typuslokalität für das Mindel zu einem prämindelzeitlichen Alter, für das er das im Rheingletschergebiet von Schreiner \& Ebel (1981) definierte Haslach diskutiert (НАввE 1985: 41). 


\section{Lagerungsverhältnisse der pleistozänen Ablagerungen auf dem Grönenbacher} Feld

Morphologisch gliedert sich das Grönenbacher Feld in zwei größere Hauptvorkommen (Abb. 2): in die Schotterfläche des Woringer Waldes vom Hof Rothmoos bis zum Schärtelesspitz (mit den gleichalten Vorkommen in seiner südlichen Verlängerung als Woringer Schotter bezeichnet) sowie in den Grönenbacher Wald westlich und südwestlich des Ortes Bad Grönenbach (Grönenbacher Schotter). Dieses Vorkommen endet im Süden ungefähr bei Herbisried, wo würmzeitliche Moränen mit unregelmäßigem Relief einsetzen.

Der Woringer Schotter fällt mit seiner Basis mit 6,9 \%o nach Norden ein. Im Bereich der kleinen Terrassenreste weiter südlich (Rechberg und Rothenstein bis Brandholz) ist bei Annäherung an die Moränen eine Versteilung auf $9,3 \%$ feststellbar. Der maximal 1,7 km breit erhaltene Woringer Schotter weist im Westen einen markanten Uferrand auf, an dem auf wenigen hundert Metern die Terrassensohle um 5 bis $10 \mathrm{~m}$ ansteigt. Vom einstigen Ufergelände haben sich die Kuppen von Hoher Rain und Kronburg erhalten, deren Schotter mit ihrer Basis um etwa 30 bis $40 \mathrm{~m}$ höher liegen. Die Mächtigkeit des Woringer Schotters beträgt 20 bis $25 \mathrm{~m}$, wobei das Material gewöhnlich karbonatisch verfestigt ist. Es ist gebankt, kornund matrixgestützte Partien wechseln sich $a b$, und zeigt den typischen Habitus glazifluviatiler Schotter. In den ehemaligen Kiesgruben $1 \mathrm{~km}$ westnordwestlich von Woringen ( $\mathrm{R} 3588980$ $\mathrm{H}$ 5310580) und bei Molzen (R $3588800 \mathrm{H}$ 5308710) konnten Messungen der Gerölleinregelungen vorgenommen werden, die eine Schüttung aus Süden belegen (Abb. 2).

Getrennt durch das Mühlbach-Tal liegt im Osten anschließend der Grönenbacher Schotter, welcher am Geländesporn der Pfaffen-Halde westlich von Grönenbach einsetzt. An der westlichen Talflanke des Mühlbaches in einer Distanz von nur ca. $400 \mathrm{~m}$ liegt die Basis des Woringer Schotters $10 \mathrm{~m}$ höher, was im Verein mit den übrigen Messpunkten und den daraus errechneten Isobasen nahelegt, dass der Grönenbacher Schotter eine eigenständige, jüngere Schüttung darstellt, die an den Woringer Schotter angelagert ist (Abb. 3). In Richtung

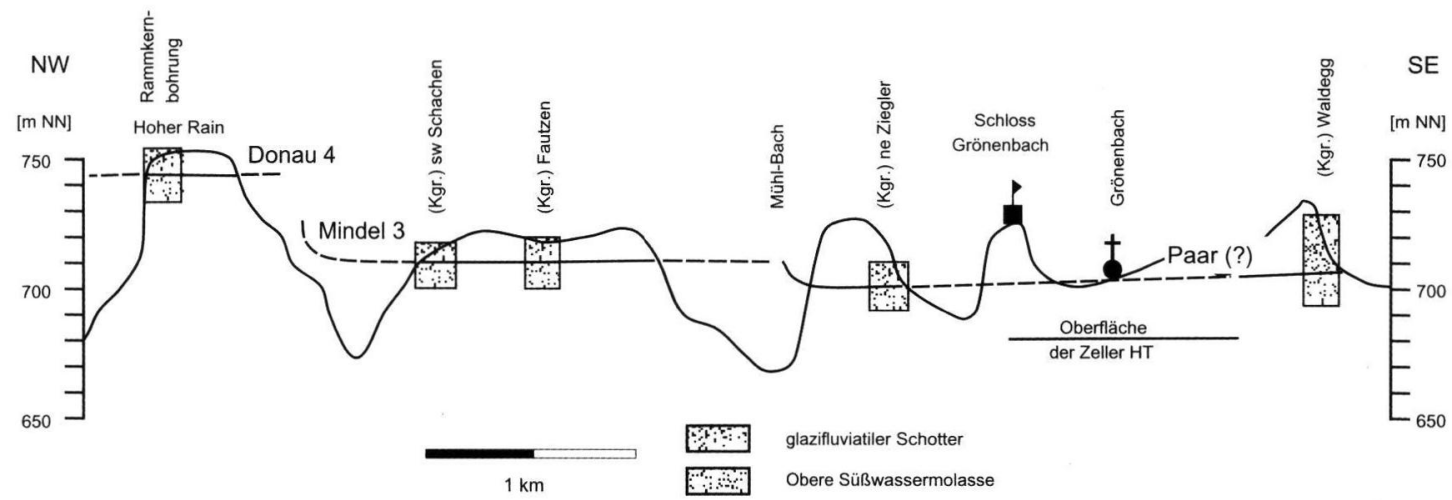

Abb. 3: Querprofil durch das Grönenbacher Feld. Erkennbar sind die Höhenunterschiede der Terrassen von etwa $10 \mathrm{~m}$ bei ansonsten nahezu ebener Schottersohle. Die Profillinie ist in Abb. 2 eingetragen.

Fig. 3: The cross section (position in Fig. 2) of the Grönenbacher Feld represents the differences in altitude of both terrace levels of about $10 \mathrm{~m}$. 
der Moränenverzahnung im Süden gleichen sich die Sohlen beider Schottereinheiten bis zu einer Höhe von $740 \mathrm{~m} \mathrm{NN}$ an. Wie aus den Daten der Abb. 2 ersichtlich ist, weist auch der Grönenbacher Schotter einen Westrand mit deutlich ansteigender Terrassensohle auf, womit insgesamt die westlichen Teile der einstigen Füllung des Illertales erhalten sind. Das Grönenbacher Feld ist damit als nach Westen ansteigende Terrassentreppe anzusehen.

Bei dem Hof Waldegg befindet sich eine aufgelassene Kiesgrube, die den Aufbau des Grönenbacher Schotters über eine Höhe von $12 \mathrm{~m}$ zeigt (Abb. 4). Die Abfolge beginnt mit einer gut 2 $m$ mächtigen Lage sehr groben, matrixgestützten Materials, das intensiv schräggeschichtet ist und eisrandnah abgelagert wurde. Häufig treten Schichten mit toniger Matrix auf, welche sich aus der Gletschertrübe abgesetzt hat und im darüberliegenden Material fehlt. Dieses besteht aus mehreren 1 bis $2 \mathrm{~m}$ mächtigen Bänken, in denen teilweise eine Verfeinerung von grobem, blockreichem Kies an der Basis bis zu Feinsandlagen am Top zu beobachten sind. Sie repräsentieren braid bars, während die zwischengeschalteten Partien aus wenig strukturiertem Kies in Rinnen abgesetzt wurden. Insgesamt werden Bedingungen eines verwilderten Flusssystems (braided river) im unmittelbaren Gletschervorfeld dokumentiert.

\section{Profil Mühlbach-Tobel}

Die räumliche Beziehung der zwei auf dem Grönenbacher Feld kartierten Terrassenniveaus wird durch die Verhältnisse im Mühlbach-Tobel westlich von Herbisried verdeutlicht, der in seinem Oberlauf die Moränenverzahnung beider Terrassen durchschneidet. An der Ostflanke des steilen Taleinschnittes ist nahe der Pumpstation der örtlichen Wasserversorgung ein ca. $12 \mathrm{~m}$ hohes Profil aufgeschlossen (Abb. 5). Die Schotterbasis wurde etwa $200 \mathrm{~m}$ weiter südlich mit $737 \mathrm{~m}$ NN eingemessen. Über Versturzmassen folgt ein Schotter mit sandigem Zwischenmittel, der über die aufgeschlossenen $5 \mathrm{~m}$ ein coarsening-up aufweist. Er wird von deutlich gröberem Material mit toniger Matrix überlagert, das als Moräne (Schottermoräne) anzusprechen ist. Schichtungsstrukturen im Material deuten auf Umlagerungsvorgänge hin. Auf dieser Abfolge von Vorstoßschotter und Moräne ist ein wenige Dezimeter mächtiger Verwitterungshorizont entwickelt, der durch rötlich-braune Farbe und einen erhöhten Tongehalt auffällt.

Die Geröllpetrographie des frischen Sediments (Kornfraktion 6,3 - $20 \mathrm{~mm}$ ) wird im Mittel durch 70\% Kalkstein, 17\% Dolomit und 13\% silikatische Komponenten geprägt. Im Schwermineralbestand (Tab. 2) dominiert der Granat mit ca. 57\%, gefolgt von metamorphen $\mathrm{Mi}$ neralen (25\%), der Epidotgruppe (15\%) und stabilen Mineralen mit durchschnittlich 3\%. Die über die Profilhöhe hinweg gleichbleibende petrographische Zusammensetzung ist auf das unveränderte alpine Liefergebiet zurückzuführen.

Die im Verwitterungshorizont enthaltenen Gerölle sind angewittert und die Dolomite verascht. Eisen- und Manganverbindungen sind in cm-mächtigen Lagen, in denen auch der Karbonatgehalt erhöht ist, angereichert. Die Matrix des Horizontes ist außerhalb dieser Lagen stark karbonatreduziert (Tab. 2). Wegen der lagigen Struktur wurden die Proben als Mischproben aus dem unteren bzw. oberen Bereich des Verwitterungshorizontes entnommen. Es ist zu berücksichtigen, dass das über- und unterlagernde Sediment zum überwiegenden Anteil aus kalkalpinen Geröllen besteht und die Nagelfluhbildung innerhalb des gesamten Profils zu sekundärer Aufkalkung geführt hat. Im Vergleich zum frischen Schotter (Proben 1 und 4) mit unter einem halben Prozent Eisen ist eine Anreicherung auf ca. 3\% Fe und 4,4\% $\mathrm{Fe}_{2} \mathrm{O}_{3}$ innerhalb des Verwitterungshorizontes 
[m NN]

725
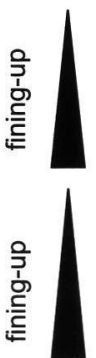

720

715

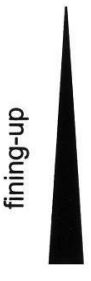

705

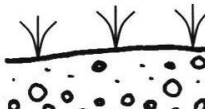

0.000000

$0 \because \because \because 0$. $\because \because \because 0^{\circ} \circ$

0 =

$\because \because \therefore \circ$

$30 \because 0$

$0: 0.0$

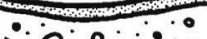

$\because 0 \% 0$

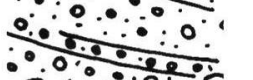

$00.00 \% 0$

00.00

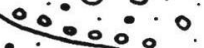

$\because \because \therefore 0$

$\therefore: 0.0$

$\because \therefore 000$

०0.00

$\therefore$ \%०.
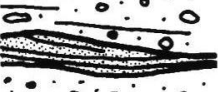

$00 \div 0$

$\because \because 0 \%$
$\because \because 0 \%$

$\because 0: 0$

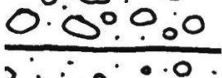

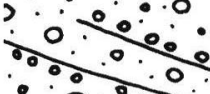

$\therefore 0.9 .00$
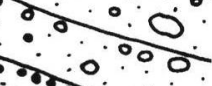

.

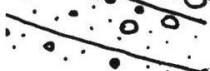

Halde

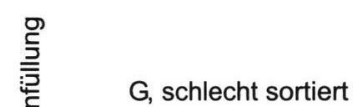

Sandlinse, schräggeschichtet

G, schlecht sortiert, massig, Nagelfluh

fS-Lage

m-gG, schwach schräggeschichtet

$x$

G, massig, wenige Sedimentstrukturen, Nagelfluh

G, X eingelagert, schlecht sortiert, massig, matrixgestützt, lagenweise korngestützt und gut sortiert, Nagelfluh

\section{fS-Lage, gut geschichtet}

G, partienweise mit toniger Matrix, schlecht sortiert, nach oben zunehmend schräggeschichtet, partienweise fining-up-Sequenzen eingeschaltet, matrixgestützt, einzelne Lagen korngestützt, Nagelfluh

f-mG, intensiv schräggeschichtet, gut sortierte Lagen mit toniger Matrix eingeschaltet, $\mathrm{X}$ mit $A=20 \mathrm{~cm}$ eingelagert, matrixgestützt, einzelne Lagen korngestützt, Nagelfluh

Schichtblätter $15^{\circ} / 310^{\circ}$

Abb. 4: Profil der (Kgr.) Waldegg innerhalb des paarzeitlichen Grönenbacher Schotters (TK 25 Blatt 8127, R 3592510 H 5304270). Über einer eisrandnahen Schüttung folgen ab einer Höhe von $715 \mathrm{~m}$ NN typische glazifluviatile Ablagerungen, deren Strukturen auf Rinnenfüllungen sowie braid bars hindeuten.

Fig. 4: Section of the old gravel pit near Waldegg with the Paar age Grönenbacher Gravel (TK 25 sheet 8127 , R 3592510 H 5304270). Proximal gravels were superimposed by typical glaciofluvial deposits representing scour fills as well as braid bars. 


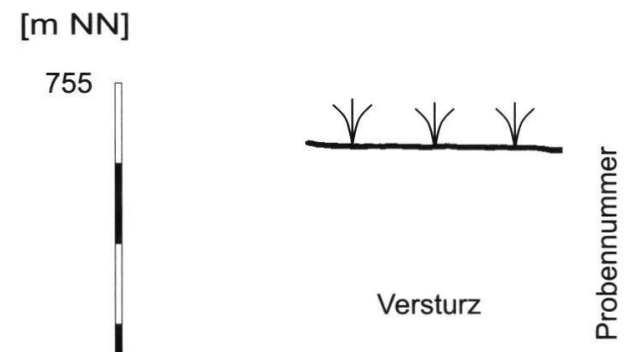

750

740

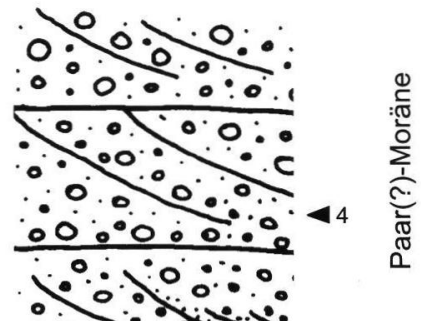

-
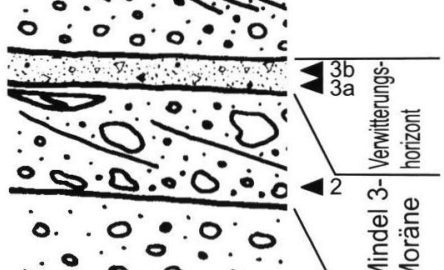

. . 0.0

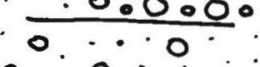

- .0 .00$.

$\therefore \div .0$.

-.......



$\therefore \frac{.}{. \cdot .}$

1

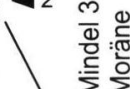

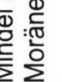
schräggeschichtet, im oberen Teil Sandlinsen, Nagelfluh, $\mathrm{HCl}^{++}$

$\mathrm{gG}$, unstrukturiert, matrixgestützt, $\mathrm{HCl}^{++}$

G, sandige Matrix, undeutlich gebankt, matrixgestützt, wenige Lagen korngestützt, unverfestigt, $\mathrm{HCl}^{++}$

$\mathrm{mG}$, gg, tonige Matrix, Nagelfluh, $\mathrm{HCl}^{++}$

m-gG, tonige Matrix, schwach schräggeschichtet, an Schichtungsblättern gut sortiert und korngestützt, sonst matrixgestützt, Nagelfluh, $\mathrm{HCl}^{+}$

m-gG, gG, tonige Matrix, an Basis 0,5 m ungeschichtet, darüber schräggeschichtet, matrix垈 lagig angereichert, Verwitterungsintensität mit

Tiefe abnehmend, $\mathrm{HCl}-$, Horizont durchziehend, stellenweise auskeilend

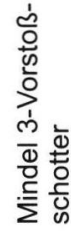
gestützt, an Schichtungsblättern korngestützt, $\sum_{\text {C }} \mathrm{fS}_{\mathrm{HCl}}{ }^{-}$, gelblich, kreuzgeschichtet, unverfestigt,

Versturz

Abb. 5: Profil des Mühlbach-Tobels (TK 25 Blatt 8127, R 3590815 H 5302220). Auf einer Vorstoßsequenz des Mindel 3-Eises mit Vorstoßschottern und Moräne ist ein gekappter Paläoboden erhalten. Er wird von einer Moräne des paarzeitlichen Illergletschers überlagert. Die Nummern der Proben (Tab. 1) sind eingetragen.

Fig. 5: Section of the Mühlbach gorge (TK 25 sheet 8127, R 3590815 H 5302220). Glaciofluvial gravels of an ice advance grade into a till of Mindel 3 age. In that sediment a 30 to $40 \mathrm{~cm}$ thick paleosol is developed overlain by a Paar age gravel till of the Illerglacier. Numbers of recovered samples (Tab. 1) are noted. 
Tab. 2: Analysendaten zum Profil im Mühlbach-Tobel. Die Probennahmepunkte sind in Abb. 5 eingetragen.

Tab. 2: Results of qualitative analyses of the samples of the Mühlbach gorge. The sample positions are given in Fig. 5.

\begin{tabular}{|c|c|c|c|c|c|c|c|}
\hline Probennr. & $\begin{array}{c}\text { Gesamt } \mathrm{CO}_{3}{ }^{2-}- \\
\text { Gehalt [\%] }\end{array}$ & \multicolumn{2}{|c|}{$\begin{array}{c}\text { Pedogenes } \\
\mathrm{Fe}\end{array}$} & $\begin{array}{c}\text { Eisen [\%] } \\
\mathrm{Fe}_{2} \mathrm{O}_{3}\end{array}$ & \multicolumn{4}{|c|}{ Schwermineralgehalt [\%] * } \\
\hline 4 & 11,4 & 0,34 & 0,48 & 58 & 15 & 25 & 2 \\
\hline $3 \mathrm{~b}$ & 6 & 3,06 & 4,37 & - & - & - & - \\
\hline $3 \mathrm{a}$ & 12 & 0,94 & 1,34 & - & - & - & - \\
\hline 2 & 19 & 0,61 & 0,88 & - & - & - & - \\
\hline 1 & 10 & 0,40 & 0,70 & 55 & 15 & 25 & 5 \\
\hline
\end{tabular}

* Epidotgr. - Epidot, Zoisit, Metam. - Hornblende, Staurolith, Sillimanit, Disthen, Andalusit, Stab. - Turmalin, Rutil, Topas

nachweisbar (Tab. 2). In den Werten zum Eisengehalt spiegelt sich eine mit der Tiefe abnehmende Verwitterungsintensität wider, die für einen insitu-liegenden Boden spricht.

Von Herrn A. Link (Geologisches Institut Köln) wurden freundlicherweise Röntgenanalysen des Tones aus der Matrix des Verwitterungshorizontes - durch Abschlämmen und Sedimentieren der Kornfraktion $<63 \mu \mathrm{m}$ gewonnen - vorgenommen. Das Spektrum wird von Calcit dominiert, der durch Aufkalkung zugeführt wurde. Der gegenüber dem frischen Sediment erhöhte Dolomitgehalt beruht auf der Veraschung von Dolomitgeröllen (chemische Verwitterung von Dolomit sensu Fezer 1969), wie sich durch die vergleichende Messung eines verwitterten Dolomites (Dolomitasche) bestätigen ließ. An Glimmermineralen ließen sich in der Reihenfolge abnehmender Häufigkeit Muskowit, Kaolinit und Montmorillonit nachweisen. Chlorit konnte durch Glühen der Proben bei ca. $600^{\circ} \mathrm{C}$ ausgeschlossen werden, die Behandlung mit Glykol brachte den Nachweis von Montmorillonit gegenüber Chlorit. Diese Befunde zeigen, dass innerhalb des Tonmineralbestandes die Verwitterungseffekte durch die spätere Aufkalkung überprägt worden sind.

Bei der Beurteilung des fraglichen Horizontes ist zu bedenken, dass in mächtigen Schotterkörpern zuweilen stark rot gefärbte Horizonte an
Schichtfugen auftreten können, die auf Eisenausfällungen durch Sickerwässer beruhen. Im Unterschied zu dem hier beschriebenen Horizont weisen die Gerölle Eisenbeläge oder dünne Verwitterungsrinden auf, sind aber ansonsten weitgehend frisch erhalten. Diese hydromorphen Bodenbildungen sind typischerweise an Materialwechsel gebunden und setzen lateral nach kurzer Distanz aus. Der Verwitterungshorizont des Mühlbach-Tobels hingegen tritt innerhalb von Moränenablagerungen auf und lässt im Geländebefund und in den Daten des Eisengehaltes eine mit der Tiefe abnehmende Verwitterungsintensität erkennen. Letzteres könnte gegen eine hydromorphe Bodenbildung oder die Deutung als laterale Verzweigung einer Verwitterungsschlotte mit Einschwemmung verwitterten Materials sprechen. In vorliegender Arbeit wird daher der fragliche Horizont als insitu-befindlicher Rest eines Bodens angesehen. Dieser Befund passt insofern in den regionalen Kontext, als dass der liegende Vorstoßschotter nebst Moränenauflage dem Woringer Schotter als älterer Einheit des Grönenbacher Feldes entspricht. Nach Deglaziation, fluviatiler Tiefenerosion und vermutlich der Ausbildung einer Verwitterungsdecke erfolgte eine erneute Eisüberfahrung des südlichen Grönenbacher Feldes bis zu der in Abb. 2 eingezeichneten Linie (Paar(?)-Eisrandlage). Von der über dem 
Tab. 3: Stratigraphische Tabelle des Illergletscher-Vorlandes mit Angabe der zugehörigen Vorkommen glazifluviatiler Terrassen im Iller- und Günztal. Vor dem Mindel 3 war das Illertal noch nicht von Schmelzwasser durchflossen, da sie über das Obergünzburger Becken abliefen. Im Riß 2 erfolgte der Abfluss nördlich Memmingen nur noch durch das Illertal.

Tab. 3: Stratigraphic system of the Illerglacier Foreland with reference to the glaciofluvial terraces occurring in the valleys of Iller and Günz. Prior the Mindel 3 stage the Iller valley was not yet used by meltwater as they flowed from the glacier basin of Obergünzburg to the north. During the Riss time north of Memmingen only the Iller valley was active.

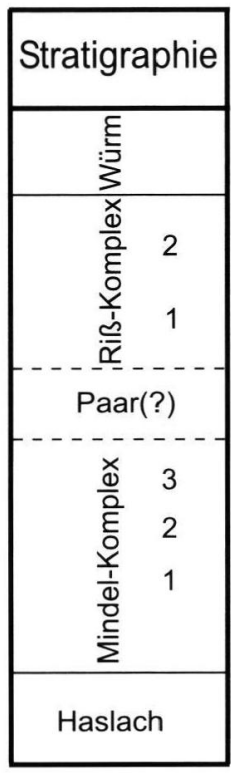

\begin{tabular}{|l|l|l|}
\hline \multicolumn{2}{|c|}{ Terrassenabfolge } \\
\hline Niederterrasse & Mindel-/Auerbachtal & \multicolumn{1}{c|}{ Illertal } \\
\hline Hawanger Feld & Niederterrasse & Memminger Feld \\
\hline $\begin{array}{l}\text { Schwaighauser Schotter } \\
\text { Ungerhauser Wald } \\
\text { Untrasr.-Stephansr. Schotter, } \\
\text { Oberegg-Saulengr. Schotter } \\
\text { Boden von Hinterschmalholz } \\
\text { Vorkommen südlich von Böhen }\end{array}$ & $\begin{array}{l}\text { Obergünzb.-Mindelheimer } \\
\text { Sch., Warmisrieder Feld } \\
\text { Hörtwald }\end{array}$ & $\begin{array}{l}\text { Stzenhofer Feld, } \\
\text { Zeller Hochterrasse }\end{array}$ \\
\hline
\end{tabular}

Verwitterungshorizont abgelagerten Moräne nimmt der Grönenbacher Schotter als jüngere Einheit seinen Ausgang.

\section{Diskussion}

Aufgrund der beschriebenen morphostratigraphischen Situation ist das Grönenbacher Feld in Übereinstimmung mit HaBBE (1986) jünger als die Vorkommen, die vom Obergünzburger Becken ausgehen. Dies wird durch Ergebnisse der 3D-Modellierungen gestützt, die hydraulische Grundparameter sowie die regionale flussgeschichtliche Entwicklung in die Rekonstruktion der Terrassenkörper einbeziehen (BE-
CKer-Haumann im Druck). An der Holzheuer Höhe nordöstlich des Ortes Ronsberg (Abb. 1) befindet sich eine markante Altmoräne, von der die Jüngeren Deckenschotter ausgehen und nach Nordwesten in das Günztal sowie nach Nordosten ins Mindeltal hineinziehen. Letztgenannter Strang geht in den KirchheimBurgauer Schotter über, der sich nach Norden bis zur Donau verfolgen lässt und von LEGER et al. (1972) aufgrund pedostratigraphischer Befunde in den Mindel-Glazialkomplex gestellt wurde. Diese Ablagerungen liegen dem interglazialen Boden von Hinterschmalholz auf (RÖGNER \& LÖSCHER 1987) und stellen die älteste mindelzeitliche Akkumulation dar (M 1). Unter anderem im Auerbachtal zwischen Markt 


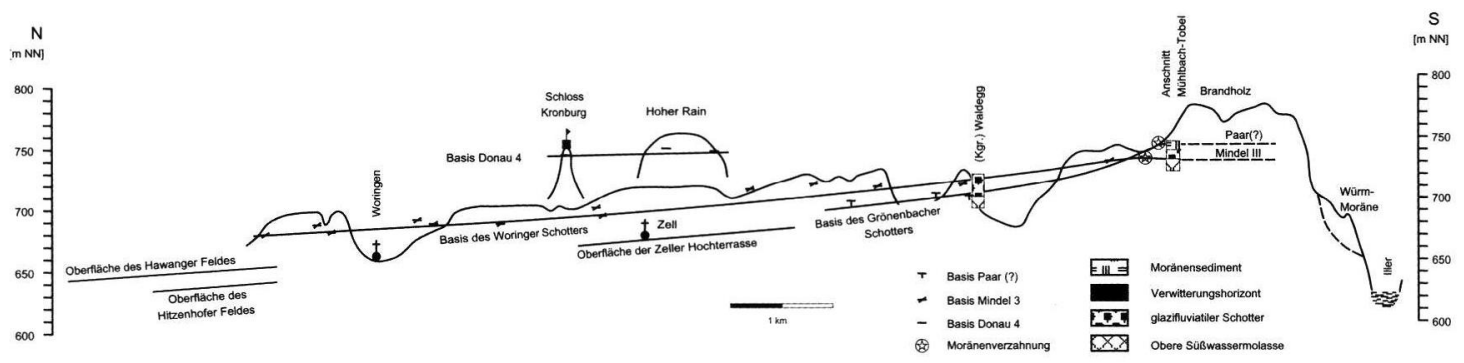

Abb. 6: Längsprofil durch das Grönenbacher Feld. Im Bereich des Mühlbach-Tobels sind zwei Moränenverzahnungen, die durch einen gekappten Paläoboden getrennt werden, entwickelt. Die Profillinie ist in Abb. 2 eingezeichnet.

Fig. 6: Longitudinal section of the Grönenbacher Feld displaying two sediment sequences of distinct ice advances seperated by a paleosol.

Rettenbach und Mindelheim finden sich zwei tiefere Niveaus von Schmelzwasserterrassen $(M$ 2, M 3, Becker-Haumann et al. 1997: 81), deren jüngstes Niveau dem Schotterstrang an der Westseite des Illertales mit den heutigen Vorkommen Woringer und Schwaighausener Schotter entspricht. Im Gesamtsystem der mindelzeitlichen Terrassen nimmt der Woringer Schotter nach vorliegendem Konzept die jüngste Position M 3 ein (Tab. 3). Zwischen diesen drei Schüttungen lassen sich keine Verwitterungsbildungen nachweisen, womit es sich jeweils um Bildungen während der Eisabschmelzphase handeln dürfte. Im Bereich des Obergünzburger Lobus war zur Mindel 1 - Zeit die Breite des Tals am größten. In dessen Füllung haben sich die nachfolgenden zwei Terrassen mit weitaus geringerer Verbreitung eingelagert.

Im heutigen Illertal stellt der Woringer Schotter die erste ausgedehnte Schmelzwasserterrasse dar, die nach der Donaueiszeit gebildet wurde. Sie wurde möglicherweise in ein von periglazialen Bächen vorgeformtes, autochthones Tal geschüttet, wie es bereits НАBBE (1986: 452) dargelegt hat. $\mathrm{Zu}$ berücksichtigen ist, dass diese Verlegung - gemessen an der heutigen Schotterverbreitung - nur den Hauptabfluss des Illergletschers betroffen hat, denn zeitgleich mit dem Entstehen des Woringer Schotters waren auch das Auerbachtal, Wörthbachtal sowie vermutlich ein Schmelzwasserabfluss bei Bad Wörishofen aktiv, wie sich aus den dortigen Vorkommen von M 2 und M 3 - Terrassen ableiten lässt.

Als nächst jüngere stratigraphische Einheit als Mindel gilt allgemein das Riss, welches im Illertal in zwei Phasen geteilt wird. Die ältere wird vom Hawanger Feld und der Zeller Hochterrasse repräsentiert, jedoch belegt das Längsprofil (Abb. 6), dass eine Verknüpfung mit dem Grönenbacher Schotter nicht gelingt. Dieser zieht mit seiner Sohle oberhalb der Oberfläche des Hawanger Feldes nach Norden und ist gemäß dem Prinzip der Terrassenstaffelung älter als dieses. Das Hawanger Feld wird als risszeitlich angesehen, was durch talgeschichtliche Zusammenhänge und Deckschichtenprofile hinreichend gesichert ist. So werden von BIBus (1995: 143-144) die Profile Benningen und Albishofen beschrieben, in denen zwei Paläoböden ein drittletzt-eiszeitliches Alter und damit eine Zuordnung der Terrassenschotter in das Riss 1 belegen.

Auf dem Grönenbacher Schotter ließ sich bislang kein Deckschichtenprofil finden, womit zu seiner Einstufung terrassenstratigraphische und geomorphologische Überlegungen herangezogen werden müssen. Auffallend ist seine 
erosiv stark zerschnittene und in Einzelvorkommen aufgelöste Verbreitung, die ihn eher zu den Jüngeren Deckenschottern als zu den Hochterrassen stellt, welche den Charakter von Talfüllungen mit noch weitgehend unversehrter Oberfläche haben. Das war bereits für PeNCK \& BrüCKNer (1909: 1161) Anlass, auf das Phänomen besonders intensiver Erosionsprozesse zwischen Mindel- und Riss-Eiszeit einzugehen (in der nachfolgenden Literatur als „Großes Interglazial" bezeichnet). Immerhin musste die Erosion lange genug wirken können, um die einstige Füllung des Illertales bis auf den Schwaighausener und Grönenbacher Schotter vollständig auszuräumen und um diese Vorkommen intensiv zu zerschneiden. Ein weiteres Indiz auf deren höheres Alter als Riss ist die Verwitterungstiefe, denn Schlotten von einigen Metern Tiefe - sie gelten als typisch für prä-risszeitliche Terrassen - lassen sich nur im Woringer und Grönenbacher Schotter stellenweise finden. Die aufgezeigten Befunde sprechen für ein prärisszeitliches Alter des Grönenbacher Schotters, was zur Problematik der Paar-Eiszeit überleitet. Die Paar-Eiszeit als eigenständige stratigraphische Einheit zwischen Mindel und Riss wurde von SCHAEFER (1951: 141) im Isar-Loisach-Gebiet eingeführt und im Illergebiet das Hawanger Feld als Stratotyp benannt (SCHAefer 1995: 176). Die Hochterrassen des Günztales gliedert er morphologisch in sechs Einheiten, die er als „Obere Hochterrassen “ zusammenfasst (Schaefer 1995: 167-174, Abb. 15) und als paarzeitlich einstuft. Das Interglazial zum Riss, das in seinem Schema durch die Hochterrassen im Rothtal überliefert ist, soll nur durch die Höhendifferenz beider Terrassensysteme von knapp $10 \mathrm{~m}$ belegt sein. Ausführlich hat HaBbe (1997) begründet, dass der Gliederungsversuch und die stratigraphische Einstufung dieser Ablagerungen aus methodischen Gründen umstritten sind und Inkonsistenzen zu Nachbargebieten bestehen. Auch die eigenen Geländeergebnisse bestätigen die Gliederung des
Grönenbacher Feldes in zehn Terrassen nicht. Zudem sind Eintiefungsbeträge innerhalb von Schmelzwassersystemen keine ausreichende Grundlage für das Postulieren von Warmzeiten zwischen kaltzeitlichen Akkumulationen, da auch Eisstände während Deglaziationsphasen sich im Gletschervorfeld in unterschiedlichen Terrassenniveaus ausprägen können.

Der Woringer Schotter des Grönenbacher Feldes wird gemäß der stratigraphischen Ergebnisse aus dem Illergletscher-Vorland in BecKerHaumann (2002) und der 3D-Modellierungen der Schmelzwasserterrassen des IllergletscherVorlandes (Becker-Haumann im Druck) dem Mindel 3 zugerechnet. Damit ist er jünger als der Maximalstand der Mindeleiszeit während des Mindel 1, der durch die Endmoräne an der Holzheuer Höhe überliefert ist, und jünger als die M 2-Terrassen. Sie finden sich im heutigen Auerbachtal und im Ungerhauser Wald östlich von Memmingen, wo Schotter etwa $35 \mathrm{~m}$ oberhalb der Vorkommen des Schwaighausener Schotters liegen. Dieser stellt die nordwärtige Verlängerung des Woringer Schotters dar.

Die Einstufung des Grönenbacher Schotters ist problematischer, da es folgende Möglichkeiten gibt:

1. Der Grönenbacher Schotter könnte als jüngste Einheit in die Mindeleiszeit (Mindel 4) gehören. Der Habitus des Grönenbacher Schotters steht dieser Deutung nicht entgegen, ebenso entspricht der Eintiefungsbetrag zwischen dem Woringer und Grönenbacher Schotter den Werten, die weiter im Osten zwischen den mindelzeitlichen Schmelzwasserterrassen erreicht werden. Der Verwitterungshorizont im Mühlbach-Tobel deutet jedoch darauf hin, dass es vor Ablagerung des Grönenbacher Schotters zu einer eisfreien Periode gekommen ist. Die rudimentäre Erhaltung des Horizontes erlaubt keine Entscheidung über die klimatische Wertigkeit der Sedimentationsunterbrechung, womit sich die Entscheidung, ob ein Interstadial oder Interglazial angezeigt wird, nicht treffen lässt. 
2. Der Grönenbacher Schotter könnte die erste Schmelzwasserakkumulation des Riss darstellen, welches damit - wie im Rheingletschergebiet (SCHreIner 1996) - dreigeteilt wäre. Es wurde oben jedoch dargelegt, dass der Verwitterungszustand des Grönenbacher Schotters deutlich höher als der der Hochterrassen und die Zertalung wesentlich intensiver ist. Daher ist von einem höheren Alter als Riss auszugehen.

3. Der Grönenbacher Schotter könnte dem Paar zugehören. Problematisch bei dieser Einstufung ist, dass das Hawanger Feld zwar von Schaefer (1995) als Typusregion benannt wurde, jedoch allgemein als Hochterrasse des Riss 1 angesehen wird. Sollte der Verwitterungshorizont im Mühlbach-Tobel ein gekappter interglazialer Boden sein, wäre der Grönenbacher Schotter in der Eiszeit, die dem Mindel folgt, entstanden. Aufgrund der morphostratigraphischen Befunde und des Verwitterungszustandes sollte die Terrasse allerdings deutlich älter als Riss sein, womit die Bezeichnung Paar infrage käme.

Wenngleich für keine der vorgestellten Möglichkeiten gegenwärtig der Beweis angetreten werden kann, wird die letzte Deutung am ehesten den Geländebefunden gerecht: Der Verwitterungshorizont wird darin als Anzeiger für eine Deglaziation mit nachfolgender warmzeitlicher Bodenbildung gewertet; der Verwitterungszustand des Grönenbacher Schotters unterscheidet ihn von den risszeitlichen Hochterrassen; die Moränenverzahnung im Süden des Grönenbacher Feldes spricht für einen eigenständigen Eisvorstoß. Auf der Basis dieser Lösung wird in Tabelle 3 die Stratigraphie des Grönenbacher Feldes entwickelt und in den Zusammenhang der regionalen Terrassenabfolge gestellt.

Bei der Diskussion der Befunde wird die Schwierigkeit offenbar, im schwäbischen $\mathrm{Al}$ penvorland stratigraphische Arbeiten gemäß der Forderungen von Murphy \& Salvador (1999) oder Steininger \& Piller (1999) durchzuführen, denn bis auf wenige Ausnahmen sind nur kaltklimatisch gebildete Sedimente überliefert.
Aufgrund der dynamischen Vorgänge im glazialen Bereich und dem Schmelzwasserge-prägten Gletschervorfeld sind warmzeitliche Bildungen oder solche mit organogenem Inhalt, der paläontologische Befunde erbringt, nur punktuell erhalten. Bei einem geschätzten Alter von 400 bis $600 \mathrm{ka}$ ist derzeit die Datierung der Sedimente mit physikalischen Methoden nicht zuverlässig möglich. Da in Schmelzwassersystemen die Vorgänge Erosion, Akkumulation und Umlagerung gleichzeitig vorkommen können, ist zudem der Zeitinhalt der Terrassenablagerungen unbekannt.

Trotz dieser Schwierigkeiten verdeutlicht das vorstehend beschriebene Beispiel, dass die Morphostratigraphie gute Informationen zur Ablagerungsreihenfolge zu erbringen vermag. Mit Hilfe dieser Methode kann eine regionale Chronologie der überlieferten geologischen Ereignisse aufgestellt werden, die sich mit Nachbargebieten korrelieren lässt. Wichtig ist die Berücksichtigung des gesamten Gletschervorlandes, da wiederholte Talverlegungen dazu geführt haben, dass aufeinanderfolgende Terrassenakkumulationen in entfernt gelegenen Teilregionen des Untersuchungsgebietes ausgebildet sind. Sofern sich datierbare Profile finden lassen, wird die regionale Chronologie zur Chronostratigraphie erweitert. Im Iller-LechGebiet ist diese Datenbasis noch so gering, dass morphostratigraphische Befunde bislang einen hohen Stellenwert behalten haben.

\section{Literaturverzeichnis}

Becker-Haumann, R. (1995): Zur Korrelation der Aindlinger Terrassentreppe mit der Mindel-Lech-Platte. - Geologica Bavarica, 99: 165-178; München.

Becker-Haumann， R. (2002): Ein neues Konzept für das drittletzte Glazial (MindelGlazial) im bayerischen Alpenvorland. - Z . Geol. Wiss., 30: 173-190; Berlin. 
Becker-Haumann, R. (im Druck): Anwendungen der Geoinformatik für die hochauflösende 3D-Modellierung quartärer Terrassenkörper - Die prärisszeitliche Chronologie und Paläogeographie des Illergletschergebietes, Bayerisches Alpenvorland. - Stuttgart (Schweizerbart).

Becker-Haumann, R., Bürger, M. \& Link, A. (1997): Zur Mehrphasigkeit der Mindeleiszeit im Schwäbischen Alpenvorland - Informationen von quartären Flußterrassen entlang des Auerbachtales.Geologisches Institut der Universität zu Köln, Sonderveröff., 114: 77-90, 3 Abb.; Köln.

Becker-Haumann, R., Aktas, A. \& BRUNNACKER, K. (2001): Erläuterungen zur Geologischen Übersichtskarte des MindelLech-Gebietes 1 : 100 000. - Geologica Bavarica, 106: 109-228; München.

Bibus, E. (1995): Äolische Deckschichten, Paläoböden und Mindestalter der Terrassen in der Iller-Lech-Platte. - Geologica Bavarica, 99: 135-164, 10 Abb.; München.

EberL, B. (1930): Die Eiszeitenfolge im nördlichen Alpenvorland. - 427 S., 19 Abb., 2 Taf., 1 Kte.; Augsburg (Filser).

Eichler, H. \& Sinn, P. (1975): Zur Definition des Begriffs „Mindel“ im schwäbischen Alpenvorland. - N. Jahrb. f. Geol. u. Pal., Mh., 1975/12: 705-718, 2 Abb.; Stuttgart.

Fezer, F. (1969): Tiefenverwitterung circumalpiner Pleistozänschotter. - Heidelb. Geogr. Arb., 24: 144 S., 90 Fig., 4 Abb., 1 Tab. Heidelberg.

HabBE, K.-A. (1985): Erläuterungen zur Geomorphologischen Karte 1:25000 der Bundesrepublik Deutschland, GMK 25 Blatt 18, 8127 Grönenbach. - 80 S., 17 Abb., 1 Kte.; Berlin.

HABBE, K.-A. (1986): Zur geomorphologischen Kartierung von Blatt 8127 Grönenbach (I) Probleme, Beobachtungen, Schlußfolgerungen. - Mitt. der Fränkischen Geogr.
Ges., 31/32: 365-479; Erlangen.

Habbe, K.-A. (1997): Zur Problematik der mittelpleistozänen Ablagerungen des nordöstlichen Rhein- und des Illergletschers. - Quartär, 47/48: 51-88, 20 Abb.; Bonn.

Jerz, H., Stephan, W., Streit, R. \& Weinig, W. (1974): Zur Geologie des Iller-MindelGebietes. - Geologica Bavarica, 74: 99-130, 2 Beil.; München.

Leger, M., Löscher, M. \& Puissegur, J. (1972): Les terrasses de la vallé de la Mindel en aval de Jettingen. - Bull. Soc. franc. Quatern., 2: 135-151; Paris.

Löscher, M. (1976): Die präwürmzeitlichen Schotterablagerungen in der nördlichen Iller-Lech-Platte. - Heidelberger Geogr. Arb., 45: IX+157 S.; Heidelberg.

Murphy, M. \& Salvador, A. (1999):

International Stratigraphic Guide - An abridged version. - Episodes, 22/4: 255271, 8 Fig., 4 Tab.;

Penck, A. \& Brückner, E. (1909): Die Alpen im Eiszeitalter. - 3 Bände, 1199 S, 156 Abb., 30 Taf., 19 Ktn.; Leipzig (Tauchnitz).

RöGNER, K. \& Löscher, M. (1987): Quartäre Sedimentations- und Verwitterungsphasen bei Hinterschmalholz (Regierungsbezirk Schwaben). - Mitt. Geogr. Ges. München., 72: 161-170; München.

Schaefer, I. (1951): Über methodische Fragen der Eiszeitforschung im Alpenvorland. - Z. dt. Geol. Ges., 102: 287-310; Hannover.

Schaefer, I. (1973): Das Grönenbacher FeldEin Beispiel für Wandel und Fortschritt der Eiszeitforschung seit Albrecht Penck.Eiszeitalter und Gegenwart, 23/24: 168200, 4 Abb., 2 Taf.; Öhringen.

Schaefer, I. (1995): Das Alpenvorland im Zenit des Eiszeitalters. - 2 Bde., 405+671 S., 41 Abb.; Stuttgart (Steiner).

Schreiner, A. (1996): Die Einführung der Haslacheiszeit und die 3-Teilung der Risseiszeit im östlichen Rheingletschergebiet 
(SW-Deutschland). - Eclogae geol. Helv., 89: 991-1005; Basel.

SCHreiner, A. \& Ebel R. (1981): Quartärgeologische Untersuchungen in der Umgebung von Interglazialvorkommen im östlichen Rheingletschergebiet (BadenWürttemberg). - Geol. Jb., A 59: 1-64, 9 Abb., 5 Tab., 3 Taf.; Hannover.

SINN, P. (1972): Zur Stratigraphie und Paläogeographie des Präwürm im mittleren und südlichen Illergletscher-Vorland. Heidelberger Geogr. Arb., 37: 153 S., 13
Abb., 11 Tab., 5 Ktn., 12 Prof., 21 Fig.; Heidelberg.

Stepp, R. (1981): Das Böhener Feld - Ein Beitrag zum Altquartär im Südwesten der Iller-Lech-Platte. - Mitt. Geogr. Ges. München, 66: 43-68, München.

Steininger, F. \& Piller, W. (1999): Empfehlungen (Richtlinien) zur Handhabung der stratigraphischen Nomenklatur. - Courier Forschungsinstitut Senckenberg, 209: 19 S.; Frankfurt. 